\title{
Simulation and Analysis of Passive Rolling Compensation of High Sea Salvage System
}

\author{
Liqun Lin ${ }^{1,2, a}$, Zhixin Chen ${ }^{1,2}$ \\ ${ }^{1}$ Fishery Machinery and Instrument Research Institute, Chinese Academy of Fishery Science, Shanghai200092, China. \\ ${ }^{2}$ Joint Research Laboratory for Deep Blue Fishery Engineering Equipment Technology, Qingdao National Laboratory for Marine Science \\ and Technology, Qingdao266237, China.
}

\begin{abstract}
Method and device of a flexible interception and salvage system was introduced in this paper. In order to study the effect of wave motion on salvage operation, we proposed a passive wave compensation scheme that utilizes a combination of variable-pitch cylinders and accumulators, and established the mathematical vibration model of the rolling motion of the salvage compensation system. With the relationships between the stiffness coefficient and the accumulator parametric of passive compensated gas-liquid system, we determined the effective compensation stiffness range through Mathematica simulation analysis. The relationship between the roll displacement of the salvage arm and the initial volume $V_{0}$ of the accumulator has been analysed. The results show that the accumulator $V_{0}$ in a certain range has a great influence on the passive compensation. However, when the volume is greater than $20 \mathrm{~m}^{3}$, the compensation effect is weakened, and tend to a certain value, irrespective of the passive system accumulator volume capacity, it does not achieve full compensation. The results have important guidance on the design and optimization of rolling passive compensation of the practical high sea salvage system.
\end{abstract}

\section{Introduction}

At present, China's salvage rescue system is better in the coastal waters or low sea conditions. They generally use close-up salvage methods, or through auxiliary work boats for salvaging. However, in the high sea conditions, personnel or work boats can't leave the mother ship to operate. Because in the process of salvaging important objects, the salvage equipment is affected by the great movement of the mother ship, causing a sharp swing of the salvage object, so that the salvage efficiency is relatively low, the safety is poor, and even the salvage operation can't be carried out [1]. Therefore, it is of great significance to develop salvage equipment with wave compensation function for high sea salvage operation efficiency and safety.

Wave compensation device is mainly divided into active and passive modes, and the compensation purpose is to effectively suppress the impact of wave on offshore operation. At present, the passive wave compensation system is relatively mature, and has been widely used in marine engineering at home and abroad, the compensation principle is almost similar [2-5]. However, the wave compensation equipment for different operation modes and compensation needs is different in structure, and there are different requirements for compensation variables, control methods and compensation precision. In this paper, we have studied a passive compensation technique for the salvage system of a marine rescue vessel, and introduced a method of flexible net salvage system .In order to improve the success rate of salvage and safety of lifting process in high sea conditions, we have presented a kind of passive composite wave compensation scheme with variable amplitude cylinder as the actuator, and established the mathematical vibration model of the rolling motion of the salvage compensation system. With the relationships between the stiffness coefficient and the accumulator parametric of passive compensated gas-liquid system, we have determined the effective compensation stiffness range through mathematica simulation analysis. The relationship between the roll displacement of the salvage arm and the initial volume $V_{0}$ of the accumulator has been analysed.

\section{Salvage and wave compensation principle}

Flexible salvage with professional salvage ship method under high sea conditions is a new integrating salvage method which based on marine fishery boom trawl technology, by using side crane boom to support and open the flexible fishing nets to salvage and lift objects [6-7]. The salvage system structure shown in Figure 1, mainly includes salvage crane, flexible nets, winch, variation cylinder, anti-shake device, tension rope, etc.

\footnotetext{
a Corresponding author: linliqun@fmiri.ac.cn
} 


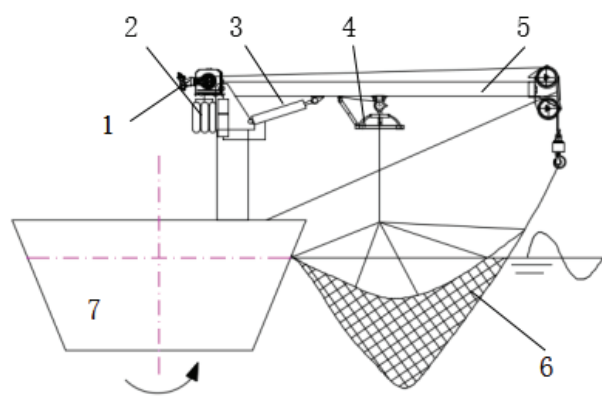

(a)

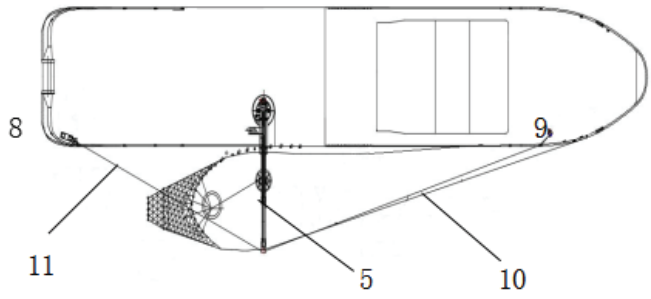

(b)

1、8、9. Winch, 2. Accumulator, 3. Variation Cylinder, 4. Anti-sway device, 5. Interception arm of salvage crane, 6 . Flexible net, 7. Salvage, 10、11. Tension rope

Figure 1 Schematic diagram of the salvage system structure

As for the motion compensation design of the salvage system, we only take into account the compensation method of the lateral rolling, according to the characteristics of its own motion and operation requirements. The passive roll compensation system consists of hydraulic cylinders, gas-liquid accumulators and air cylinders. We propose a gas-liquid passive compensation system, the hydraulic cylinder is used to bear the weight of the load, the accumulator is used to store and release the ship movement energy. The rodless cavity of the cylinder is connected with the liquid chamber of the gas-liquid accumulator, and the cylinder rod is connected to the atmosphere or the tank which pressure is the same as the atmospheric pressure, and the gas chamber of the accumulator is connected with the air bottle. In order to make the size of the compensation cylinder is not too large, the system pressure is not too high, two compensation cylinders are designed. When the ship is stationary in the equilibrium position, or the compensation system is locked to work, the compensation lift generated by the hydraulic cylinder is equal to the total weight of the salvage system. The torque of the hydraulic cylinder is equal to the total load torque of the salvage system, and the working pressure of the system depends on the total load torque. When the ship counterclockwise swing, the fixed base of the salvage crane along with the ship rose, but the interception arm of the crane (connected with the piston rod of the compensating cylinder) has the tendency to stay in the balance position due to the inertial action, the oil in the rodless chamber is compressed into the accumulator and the gas in the accumulator is compressed. Otherwise, in the rodless chamber, the oil is sucked in from the accumulator, the gas in the accumulator expands, compensating for the falling displacement and releases energy, thus achieving roll compensation. It can be seen that the compensating power of the passive compensation system is derived from the waves and the system does not need to provide additional power.

\section{Mathematical model}

\subsection{Assumption}

The salvage system is a spring damping system. According to the actual situation, the actual factors are simplified as the following basic assumptions.

(1) The wave is regarded as sinusoidal waves, the ship rolling with the wave during salvaging, $\alpha_{0}$ is roll angular displacement of the salvage ship, $\alpha_{1}$ is the interception arm's roll angular displacement, $x$ is the cylinder displacement. Do not consider the reaction force of the salvage device to the ship.

(2) The base of the salvage crane, rodless side of the cylinder, the accumulator are seen as rigid bodies following the boat movement, we do not discussed the force of the rigid bodies as a sinusoidal roll excitation for the interception arm, its motion excitation function is $\alpha_{0}=\alpha_{m} \sin (2 \pi t / T)$.

(3) The oil in the cylinder and the gas in the accumulator are regarded as massless substance, the stiffness coefficient is $k$, the viscosity resistance coefficient is $c$.

(4) The compensation system is divided into two parts, that is, intercepting salvage compensation and lifting compensation. The former mainly maintains the relative displacement of the intercept arm and the sea to keep the best intercept net shape, the latter is mainly to ensure the lifting safety. This article only consider the intercepting salvage compensation, so as no need to consider the lifting load. Regardless of the wind load on the interception arm.

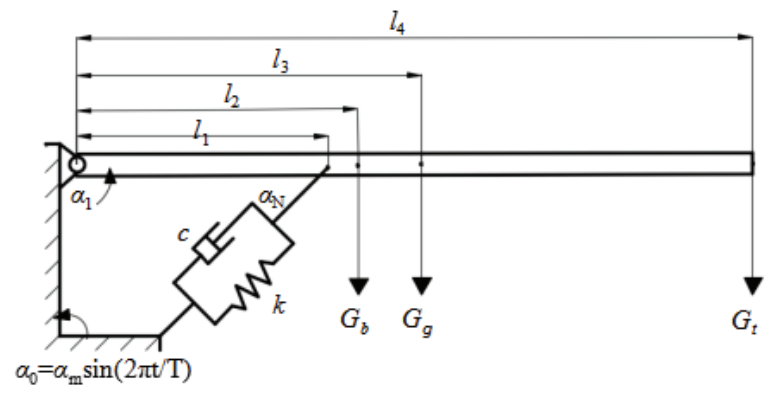

Figure 2 Mathematical model of the interception arm rolling compensation system

\subsection{Mathematical model of salvage system}

Through the above assumptions and simplification, the interception arm rolling compensation system can be simplified as shown in Figure 2, the intercept salvage system compensation dynamics equation is as:

$$
J \ddot{\alpha}_{1}+c\left(\dot{\alpha}_{1}-\dot{\alpha}_{0}\right) \sin ^{2} \alpha_{N} l_{1}^{2}+k\left(\alpha_{1}-\alpha_{0}\right) \sin ^{2} \alpha_{N} l_{1}^{2}=0(1)
$$


It is simplified as follows:

$$
\begin{aligned}
& J \ddot{\alpha}_{1}+c \sin ^{2} \alpha_{N} l_{1}^{2} \alpha_{1}+k \sin ^{2} \alpha_{N} l_{1}^{2} \alpha_{1} \\
& =\frac{2 \pi}{T} \alpha_{m} c l_{1}^{2} \cos \left(\frac{2 \pi}{T} t\right)+\alpha_{m} k l_{1}^{2} \sin \left(\frac{2 \pi}{T} t\right)
\end{aligned}
$$

Where, $J$ is total inertia of intercept arm, anti-sway device and net weight, $J=m_{b} l_{2}^{2}+m_{g} l_{3}^{2}+m_{t} l_{4}^{2}, m_{\mathrm{b}}$ is the interception arm weight, $\mathrm{kg} . m_{\mathrm{g}}$ is lifting anti roll device, weight, $\mathrm{kg} . m_{\mathrm{t}}$ is the weight of the nets, $\mathrm{kg} . l_{1}$ is the distance between hinge joint of the cylinder and the point $\mathrm{O}, \mathrm{m} . l_{2}$ is the intercept arm inertial radius, m. $l_{3}$ is distance between anti sway device installed and the point $\mathrm{O}, \mathrm{m} . l_{4}$ is distance from the points of nets on intercept arm to point $\mathrm{O}$, equal to the length of intercept arm, $\mathrm{m}$. T represents the ship's rolling period with the wave, s.Let

$$
H=\frac{\sin ^{2} \alpha_{N} l_{1}^{2}}{J}
$$

From equation (2), we can know that the salvage system inherent frequency $\omega_{\mathrm{n}}$, relative resistance coefficient $\xi$, ship roll vibration frequency $\omega$ and frequency ratio $\lambda$ are respectively as follows

$$
\begin{aligned}
& \omega_{n}=\sqrt{\frac{k}{J}} l_{1}=\sqrt{k H} \\
& \zeta=\frac{c}{2} \sqrt{\frac{H}{k}} \\
& \omega=\frac{2 \pi}{T} \\
& \lambda=\frac{\omega}{\omega_{n}}
\end{aligned}
$$

Turn the equation $(4) \sim(7)$ into equation (2) as:

$$
\begin{aligned}
& \ddot{\alpha}_{1}+2 \zeta \omega_{n} \dot{\alpha}_{1}+\omega_{n}{ }^{2} \alpha_{1} \\
& =H k \alpha_{m} \sin (\omega t)+H c \omega \alpha_{m} \cos (\omega t)
\end{aligned}
$$

Equation (8) can be solved by the complex method [8]. By using the linear superposition principle, it is obtained.

$$
\begin{aligned}
\alpha_{1} & =\frac{H k \alpha_{m}}{\omega_{n}^{2}} \frac{1}{\sqrt{\left(1-\lambda^{2}\right)^{2}+(2 \zeta \lambda)^{2}}} \sin \left(\omega t-\theta_{1}\right) \\
& +\frac{H c \omega \alpha_{m}}{\omega_{n}^{2}} \frac{1}{\sqrt{\left(1-\lambda^{2}\right)^{2}+(2 \zeta \lambda)^{2}}} \cos \left(\omega t-\theta_{1}\right) \\
& =\alpha_{m} \sqrt{\frac{1+(2 \zeta \lambda)^{2}}{\left(1-\lambda^{2}\right)^{2}+(2 \zeta \lambda)^{2}}} \sin \left(\omega t-\theta_{1}+\theta_{2}\right)
\end{aligned}
$$

Where $\theta_{1}=\arctan \left[2 \zeta \lambda /\left(1-\lambda^{2}\right)\right], \theta_{2}=\arctan (2 \zeta \lambda)$. The salvage crane interception arm rolling amplitude $B_{0}$ is:

$$
B_{0}=\alpha_{m} \sqrt{\frac{1+(2 \zeta \lambda)^{2}}{\left(1-\lambda^{2}\right)^{2}+(2 \zeta \lambda)^{2}}}
$$

The amplitude magnification factor of the roll angle displacement $\beta$ is:

$$
\beta=B_{0} / \alpha_{m}=\sqrt{\frac{1+(2 \zeta \lambda)^{2}}{\left(1-\lambda^{2}\right)^{2}+(2 \zeta \lambda)^{2}}}
$$

\subsection{Mathematical model of gas liquid passive compensation system}

During the expansion or retraction of the hydraulic cylinder of the passive compensation device, the gas is compressed or expanded by the free piston of the accumulator to change the inner volume of the cylinder, causing the pressure inside the accumulator to change. Passive compensation cylinder and accumulator system model shown in Figure 3.

Ignoring the accumulator piston mass and oil compression, compensation cylinder and accumulator pressure equal. According to the ideal gas state equation, the definition of the gas spring stiffness coefficient can be obtained as.

$$
\begin{aligned}
& p_{0} V_{0}^{n}=p_{1} V_{1}^{n} \\
& V_{1}=V_{0}-A_{a} x_{\max } \\
& k x_{\max }=\left(p_{1}-p_{0}\right) A_{a}
\end{aligned}
$$

Where, $V_{1}$ is the minimum gas volume of the accumulator cylinder, $\mathrm{m}^{3} . V_{0}$ is the initial gas of the accumulator, $\mathrm{m}^{3} . P_{1}$ is the pressure in the accumulator when the gas volume turns to $V_{1}, \mathrm{MPa} . P_{0}$ is the pressure in the accumulator when the gas volume turns to $V_{0}, \mathrm{MPa}$. $x_{\max }$ is the maximum displacement of the accumulator piston, $\mathrm{m}$. $\mathrm{n}$ is Gas polytropic index, due to its short heat exchange time, it can be considered as an adiabatic process, and the polytropic index $\mathrm{n}=1.4 . A_{\mathrm{a}}$ is the accumulator piston area, $\mathrm{m}^{2} . \mathrm{k}$ is the stiffness coefficient, $\mathrm{kN} / \mathrm{m}$. The stiffness coefficient $\mathrm{k}$ can be solved by equation (12)-(14):

$$
k=\frac{p_{0} A_{a}}{x_{\max }}\left[\left(\frac{V_{0}}{V_{0}-A_{a} x_{\max }}\right)^{n}-1\right]
$$

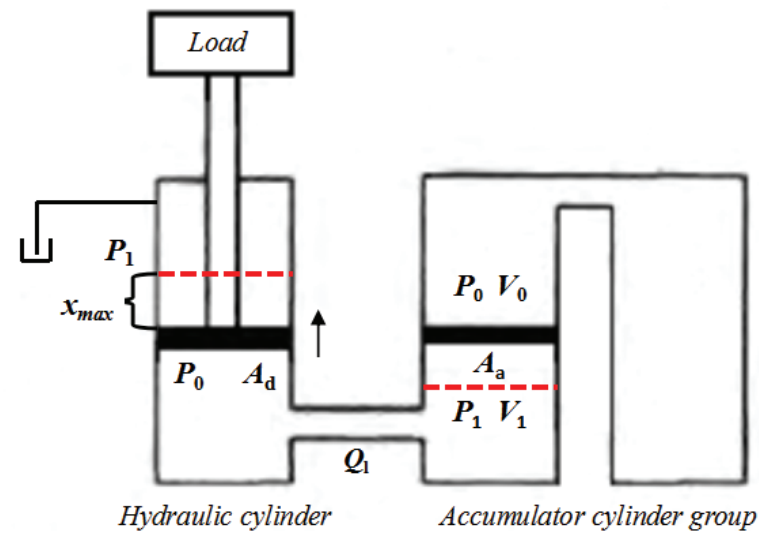

Figure 3 Passive compensation model for gas and liquid systems

\subsection{Design requirements}


In high sea conditions, the ship roll amplitude $\alpha_{\mathrm{m}}=15^{\circ}$, The period $T=10 \mathrm{~s}$. Interception arm roll angle is designed within $\pm 8^{\circ}$, other relevant parameters are as follows:

Table 1 Calculation conditions

\begin{tabular}{ccc}
\hline No. & Parameters & Value \\
\hline 1 & $G_{\mathrm{b}}$ & $90 \mathrm{kN}$ \\
2 & $G_{\mathrm{g}}$ & $12 \mathrm{kN}$ \\
3 & $G_{\mathrm{t}}$ & $10.6 \mathrm{kN}$ \\
4 & $l_{1}$ & $4.8 \mathrm{~m}$ \\
5 & $l_{2}$ & $5.6 \mathrm{~m}$ \\
6 & $l_{3}$ & $8.8 \mathrm{~m}$ \\
7 & $l_{4}$ & $16.8 \mathrm{~m}$ \\
8 & $\alpha_{\mathrm{N}}$ & $26^{\circ}$ \\
9 & $\mathrm{c}$ & $1700 \mathrm{~N} /(\mathrm{m} / \mathrm{s})$ \\
10 & $P_{0}$ & $16 \mathrm{MPa}$ \\
11 & $x_{\max }$ & $0.6 \mathrm{~m}$ \\
12 & $A_{\mathrm{a}}$ & $0.126 \mathrm{~m}^{2}$ \\
\hline
\end{tabular}

\section{Simulation results and analysis}

The rolling amplitude $B_{0}$ of the intercepting arm depends on the amplitude $\alpha_{\mathrm{m}}$ of the supporting mother ship, the frequency ratio $\lambda$ and the relative resistance coefficient $\xi$. The effect of the frequency ratio $\lambda$ and the relative resistance coefficient $\xi$ on the amplification factor $\beta$ is shown in Figure 4. It shows that when $\lambda<1$, the interceptor salvage arm roll amplitude is greater than the mother ship roll amplitude. When $\lambda=1$, that is, when the vibration frequency of the interceptor arm is equal to the frequency of the ship rolling, the interception arm resonates, the amplification factor $\beta$ increases greatly with the decrease of the relative resistance coefficient $\xi$. However, when $\lambda=\sqrt{2}$, No matter what the relative resistance coefficient $\xi$ value is, amplification factor $\beta$ is equal to 1 , that means the amplitude of the intercepting arm is equal to the ship amplitude. When $\lambda>\sqrt{2}, \xi<1$, the amplitude of the intercepting arm is less than $B_{0}$. As the frequency ratio increases, the amplification factor approaches 0 , and the larger the relative resistance coefficient is, the smaller the amplitude is. From the above analysis, in order to make the amplitude of the intercepting arm lower than the mother roll, we need to design the frequency ratio and resistance ratio of the system reasonably, satisfy this inequality $\lambda>\sqrt{2}$.

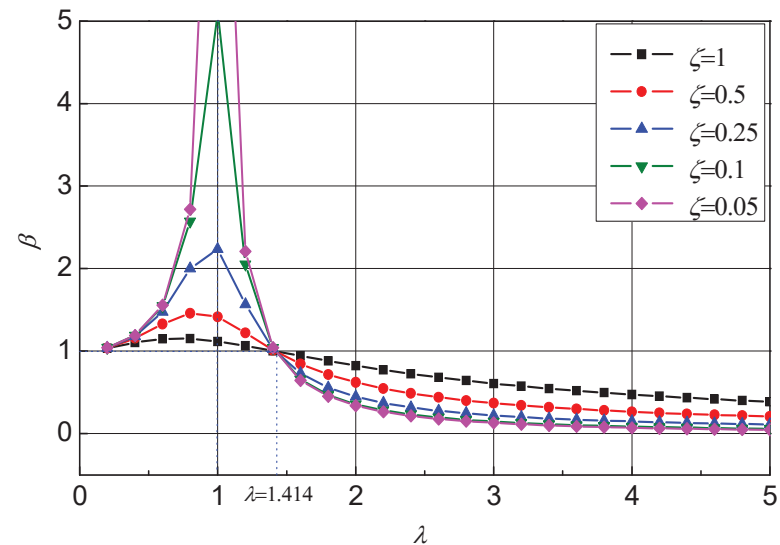

Figure 4. Relationship of $\beta-\zeta-\lambda$

The simulation curve of the interception arm is shown in Figure 5, corresponding to the initial volume of the accumulator $V_{0}=8 \mathrm{~m}^{3}, 13 \mathrm{~m}^{3}, 20 \mathrm{~m}^{3}$. After the installation of gas-liquid passive compensation, the interception arm roll angle amplitude was significantly lower than the salvage ship's roll angle amplitude. In the different initial volume conditions, the amplitude of the roll is significantly different. When $V_{0}=8 \mathrm{~m}^{3}$, the interception arm roll angle amplitude is $10^{\circ}, V_{0}=13 \mathrm{~m}^{3}$, the interception arm roll angle amplitude is $5^{\circ}, V_{0}=20 \mathrm{~m}^{3}$, interceptor arm roll angle amplitude is $2.5^{\circ}$.

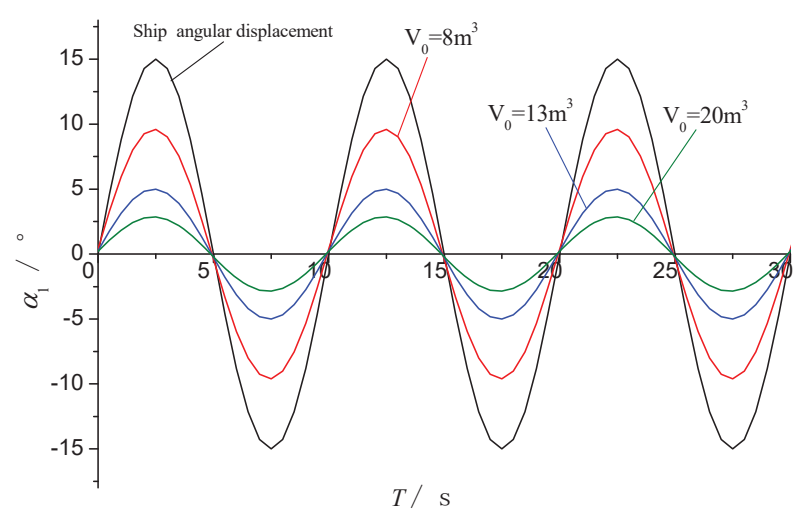

Figure 5. Passive roll compensation curve

The relationship between the compensation effect and the initial volume is shown in Figure 6. It shows that passive compensation system with accumulator is not necessarily compensated. When the accumulator volume $V_{0}$ is less than $6.5 \mathrm{~m}^{3}$, the angle amplitude of the interceptor arm is greater than $15^{\circ}$, the compensation effect is negative, which can't be compensated. When the accumulator volume $V_{0}$ is greater than $6.5 \mathrm{~m}^{3}$, the system compensation is shown to be effective. In this range, increasing the capacity of the accumulator can effectively improve the compensation effect, but the improvement of the compensation effect is not proportional to the $V_{0}$. As the volume increases, the compensation effect increases rapidly, and then the change gradually tends to be gentle, and finally tends to a certain value. However, no matter how large the volume of the passive system accumulator is, it does not achieve complete compensation. 


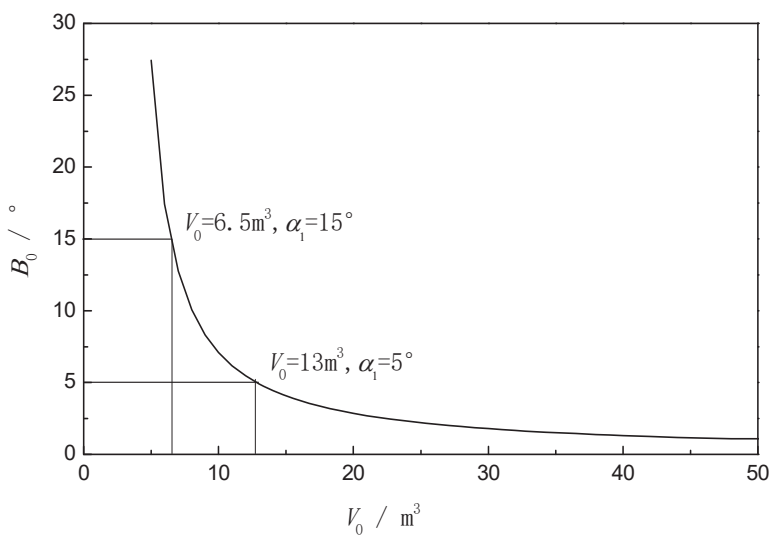

Figure 6. Curve of intercepting arm roll amplitude changing with accumulator volume

\section{Conclusion}

Aiming at ship salvage under high sea conditions, we introduced method and device based on flexible interception and salvage, and proposed a passive wave compensation scheme that utilizes a combination of variable-pitch cylinders and an accumulators, and established the mathematical vibration model of the rolling motion of the salvage compensation system. With the relationships between the stiffness coefficient and the accumulator parametric of passive compensated gasliquid system, we determined the effective compensation stiffness range through Mathematica simulation analysis. The relationship between the roll displacement of the salvage arm and the initial volume $V_{0}$ of the accumulator has been analysed. Results show that increasing the capacity of the accumulator can effectively improve the compensation effect, but the improvement of the compensation effect is not proportional to the $V_{0}$. As the volume increases, the compensation effect increases rapidly, and then the change gradually tends to be gentle, and finally tends to a certain value. However, no matter how large the volume of the passive system accumulator is, it can't achieve complete compensation. The results have important guidance on the design and optimization of rolling passive compensation of the practical high sea salvage system.

\section{Acknowledgments}

This work was financially supported by two project, one is Qingdao Ocean Science and Technology National Laboratory Aoshan Science and Technology Innovation Project No.2016ASKJ10, another is Chinese Academy of Fishery Sciences Basic Scientific Research Project No.2016YJS011.

\section{References}

1. Zhenggu $\mathrm{Xu}$, Zhixin Chen, Zhongxin Wang, et al. Equipment for salvaging floating objects [P]. CN200410024783.3 (2005)

2. Lumeng Huang, Yanting Zhang, Lei Zhang, et al. Semi-active drilling drawworks heave compensation system [J]. Petroleum Exploration and Development, 40 (5), p 620-624 (2013)

3. Haibo Wang, Qingfeng Wang. Design and Internal Model Robust Control of Underwater Towed Heave Compensation System [J]. JOURNAL OF MECHANICAL ENGINEERING, 46 (8), p 128-132 (2010)

4. Xiaoyan Tang, Shaojun Liu, Gang Wang. Modeling of heave compensation system for deep-ocean mining and its simulation of fuzzy logical control[J].JOURNAL OF CENTRAL SOUTH UNIVERSITY, 39 (1), p 128-134 (2008)

5. Jinbo $\mathrm{Wu}$, Yucheng Song. Design and Analysis of Active Heave Compensation System for Offshore Cranes [J]. China Mechanical Engineering, 27 (15), p 1989-1995, 1996 (2016)

6. Zhixin Chen, Tong Zhou HaoXu. Electric hydraulic integration controlling system for salvage equipment of aircraft returning cabin in terrible ocean environments [J]. FISHERY MODERNIZATION, 34 (6), p 55-59 (2007)

7. Liqun Lin, Zhixin Chen, Ping Liu,et al. Experimental study on high sea floating object fishing net [J].FISHERY MODERNIZATION, 43 (5), p 34-39 (2016)

8. Zhenghua Ni, Wenliang Chen, Liqun Chen.Vibration mechanics [M]. Higher Education Press (1998) 